EPJ Web of Conferences 114.02076 (2016)

DOI: $10.1051 /$ epjconf/201611402076

(C) Owned by the authors, published by EDP Sciences, 2016

\title{
Numerical and experimental study on the flow distribution in a water manifold
}

\author{
Gwansik Min ${ }^{1, a}$, Pil Jong Lee ${ }^{1}$ and Jong Hoon Kang ${ }^{1}$ \\ ${ }^{1}$ Technical Research Laboratories, POSCO, Pohang 37859, South Korea
}

\begin{abstract}
This study presents water distribution analysis of the device for spraying cooling water through specific nozzles numerically and experimentally. Numerical analysis was performed using the 3D incompressible, multi-phase flow model, for different Reynolds numbers of $4 \times 10^{5}, 8 \times 10^{5}$. Experimental analysis was performed at real-size, under the same conditions. The calculated results and the measured results for the distribution of flow were matched relatively well. The distribution of the nozzle flow depends on the Reynolds number.
\end{abstract}

\section{Introduction}

Thermal management systems that require very high heat transfer cooling with a simple equipment use the free impinging jets of liquid [1]. Run-out table temperature control is one of the processes to obtain the mechanical properties of a steel strip employed in the metal processing industry. Accelerated cooling has been implemented in hot strip mills to reduce the duration of this process in order to achieve the required coiling temperature and to obtain the desirable grain structure [24]. In general, impinging circular water jets are used as a rapid cooling system for hot steel plates [1].

Impinging water jets require the uniform mass flow rate to make strips with uniform properties. In two-phase flow, mal-distribution of the mass flow rate can significantly increase the various defects of the strip.

$\mathrm{Wu}$ and Webb [5] conducted tests in an evaporator with a horizontal header and an upward tube configuration. Lalot et al. [6] investigated fluid maldistribution in an electrically-heated heat exchanger. They developed a numerical model to determine the effectiveness of the heat exchanger and noted a 7\% reduction in the calculated heat exchanger performance due to mal-distribution in the counter-current flow heat exchangers and condensers.

Rao et al. $[7,8]$ evaluated the thermal performance of falling film plate condensers with flow mal-distribution from the feeding tube to the channel. The heat transfer coefficient was considered as a function of mass flow rate inside the channels. They evidenced a heat transfer coefficient reduction due to mal-distribution among the parallel channels.

Marchitto et al. [9] commented that uneven two-phase flow distribution may occur inside each channel, owing to the asymmetrical parallel and diagonal flow, as well as inside the header, owing to the separation of the twophase mixture.

This work presents the water distribution analysis of the manifold and comparison on experimental and numerical results. Figure 1. shows the manifold geometry considered in this paper.

\section{Approach of problem}

\subsection{Experimental setup}

Experiments were performed in a real-size test in order to investigate water distribution problems of the model manifold under various flow conditions in addition to the effect of baffle fitting.

An apparatus was made that consisted of a water manifold, an elevated tank, a pump, and a basin. The elevated tank was installed at $30 \mathrm{~m}$ height and fed the basin water through the pump. The downstream of the elevated tank flowed through a pipe with an inner diameter $(D)$ of $0.13 \mathrm{~m}$. The water manifold was connected to the flow system by a horizontal pipe with a flow meter and pressure transmitter. The instrumentation was designed to record the evolution of the flow rate and pressure inside the pipe. An array of the valves upstream of the manifold could be operated in order to control the flow rate coming from the elevated tank. The pressurized water manifold sprayed the water back into the basin. A schematic diagram of the flow system is shown in Figure 2.

The manifold consists of a horizontal channel of rectangular cross-section, two air vent halls and a system of laminar jet nozzles. The total length of the channel is

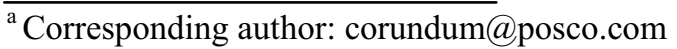




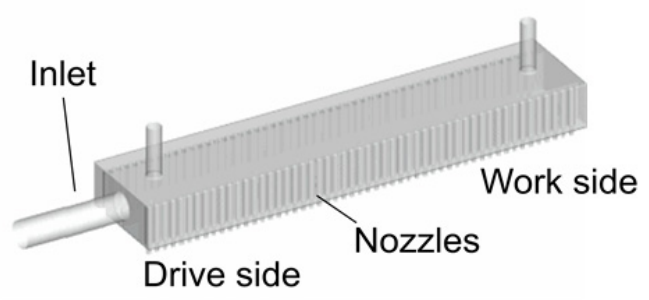

Figure 1. The model water manifold considered in this paper.

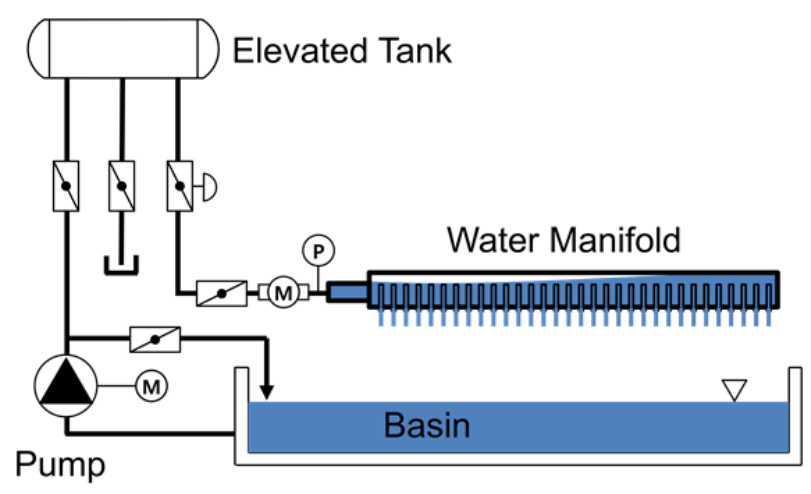

Figure 2. Schematic diagram of the flow system.

over $15 D$ and minimum cross-sectional area $(A)$ is 0.089 $\mathrm{mm}^{2}$. The gap between the nozzle and manifold wall is over $0.2 D$.

For this case, the Reynolds number is defined as follows:

$$
\operatorname{Re}=\frac{\rho V D_{\mathrm{h}}}{\mu}
$$

where, $\rho$ represents the density, $V$ the mean velocity in the minimum cross-sectional area of the manifold, $D_{\mathrm{h}}$ the hydraulic diameter calculated from the cross-section of the manifold, and $\mu$ the viscosity.

The nozzle flow rate was measured by the weight of water sprayed from the bottom of the manifold, and the measured time was over 40 seconds. The measuring points were located on the drive side, center side and work side.

\subsection{Numerical simulation}

\subsubsection{Governing equations}

The mass and momentum conservations for the incompressible multi-phase flow can be written as follows:

$$
\begin{gathered}
\nabla \cdot \mathbf{u}=0 \\
\rho \frac{\partial \mathbf{u}}{\partial t}+\rho \nabla(\mathbf{u u})=-\nabla p+\mu \nabla^{2} \mathbf{u}+\mathbf{F}
\end{gathered}
$$

where $\rho$ represents the density, $\mathbf{u}$ the velocity vector, $p$ the local pressure, $\mu$ the viscosity and $\mathbf{F}$ any body forces acting on the fluids. The momentum equation (3) is
Table 1. Numerical parameters.

\begin{tabular}{|c|c|}
\hline Parameters & \\
\hline Multiphase model & $\begin{array}{c}\text { Volume of Fluid, } \\
\text { Multi-fluid VOF (Eulerian) }\end{array}$ \\
\hline Turbulent model & SST k- $\omega$ \\
\hline $\begin{array}{c}\text { Pressure-Velocity } \\
\text { coupling scheme }\end{array}$ & SIMPLE \\
\hline Working fluid & Water, Air \\
\hline Inlet condition & Uniform velocity inlet \\
\hline Outlet condition & Constant pressure outlet \\
\hline
\end{tabular}

dependent on the volume fractions of all phases through the properties $\rho$ and $\mu$.

In the two-phase model, two types of model were compared, VOF (volume of fluid) and Multi-fluid VOF.

In the VOF model, a single set of conservation equations is shared by the phases and the volume fraction of each of the phases is tracked in each computional cell thorughtout the domain [12]. Therefore VOF adopted surface-tracking techniques and the interfacial forces are replaced by smoothly varying volumetric forces.

The multi-fluid VOF model couple the VOF model and Eulerian multi-phase models. This allows the discretization schemes suited to both sharp and dispersed interface regimes while overcoming some limitations of the VOF model that arise due to the shared velocity.

For the numerical computations, the commercial CFD code ANSYS FLUENT 15.0 was used. A QUICK scheme for the spatial discretization and SIMPLE algorithm for the pressure-velocity coupling were adopted [11]. The turbulence model is considered an SST $k-\omega$ model. Numerical convergence criterion for the residuals was $1.0 \times 10^{-3}$ for the mass components and the other quantities.

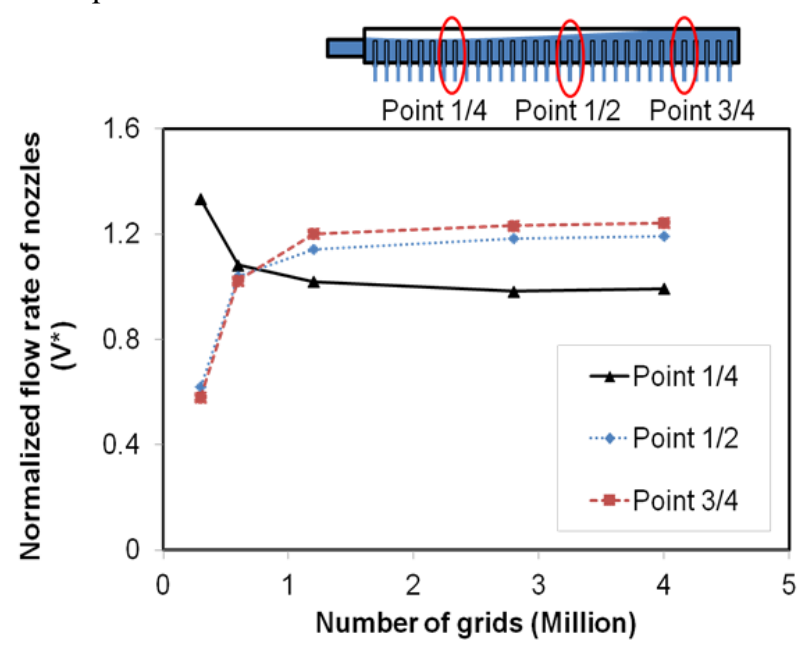

Figure 3. Normalized flow rate of simulation vs. number of grids. 


\subsubsection{Grid dependency}

In order to determine a suitable number of grids, a preliminary grid dependency test for the geometry of the manifold was carried out to verify that the solution was grid independent. Five grids with numbers of cells that ranged from 0.3 million to 4.0 million were tested.

The normalized flow rate of nozzles depending on the number of grid shown in Figure 3. We selected 1.2 million cells for the grid system. In this case, the $y+$ near the wall was 51 .

\section{Results and Discussion}

Figure 4. shows flow distribution of the water manifold from the experimental and numerical results at the $\mathrm{Re}=8.4 \times 10^{5}$. Experimental results are represented by the points; computational results are represented by the line. Single-phase flow calculation results did not describe the unbalanced flow rate distribution. In the case of the VOF model, mal-distribution was exaggerated. The Eulerian model that was calculated separately for each phase tends to be relatively consistent with the experimental results.

To compare the above two multi-phase models, the VOF model was suitable for finding the unbalanced area and the Eulerian model was appropriate for estimation of mal-distribution flow

To solve a simpler problem, the VOF model is a better choice, since it solves a smaller number of equations which requires less computational effort than the Eulerian model. If accuracy is more important than computational effort, the Eulerian model is a better choice.

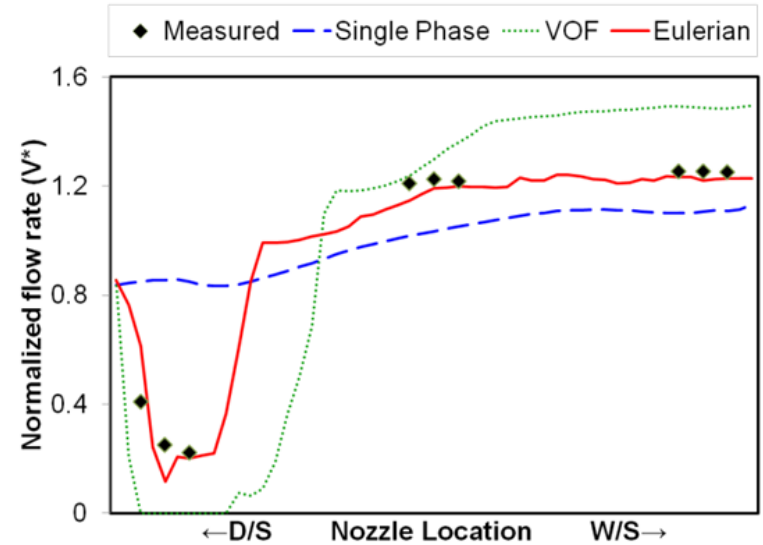

Figure 4. Comparison between the experimental and the numerical results.

The base model and modified model with slope baffles of the water manifold are shown in Figure 5. Computations and experiments were conducted for $\mathrm{Re}=$ $8.4 \times 10^{5}$, and only computations for $\operatorname{Re}=4.2 \times 10^{5}$.

The effect of slope baffles on the water distribution is shown in Figure 6. For $\mathrm{Re}=8.4 \times 10^{5}$, the model with the baffles had a better distribution compared to the base model. For $\mathrm{Re}=4.2 \times 10^{5}$, the flow rate largely changed according to the location of baffles.
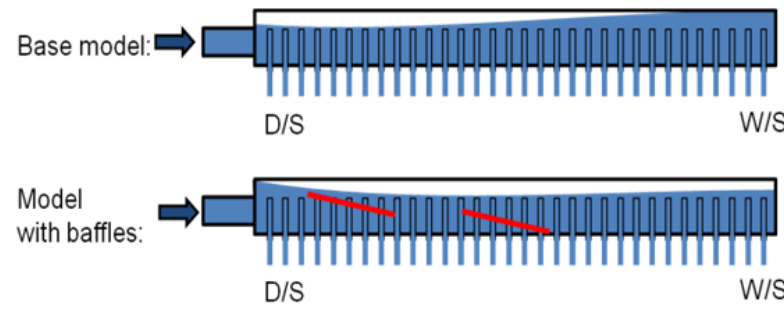

Figure 5. Schematic diagram of the base model and model with
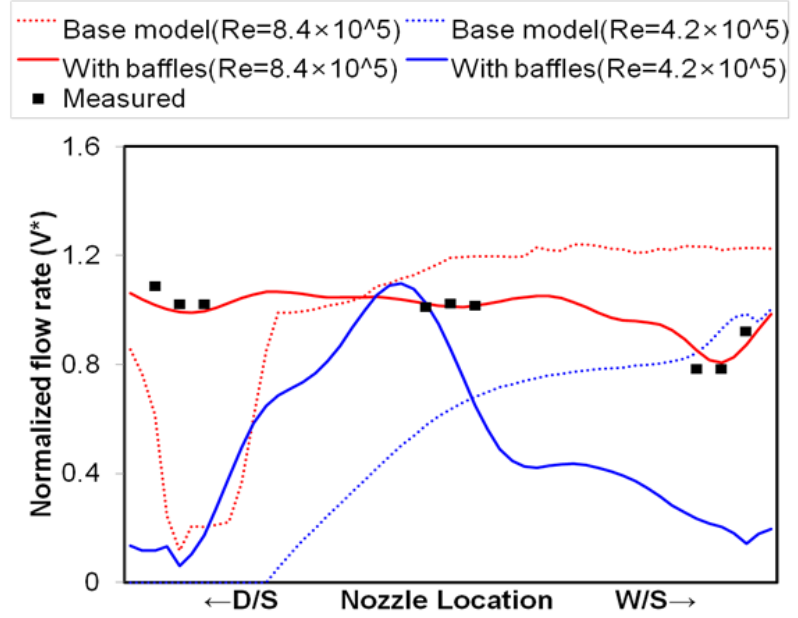

Figure 6. Water distribution comparison between base model and model with the baffles.

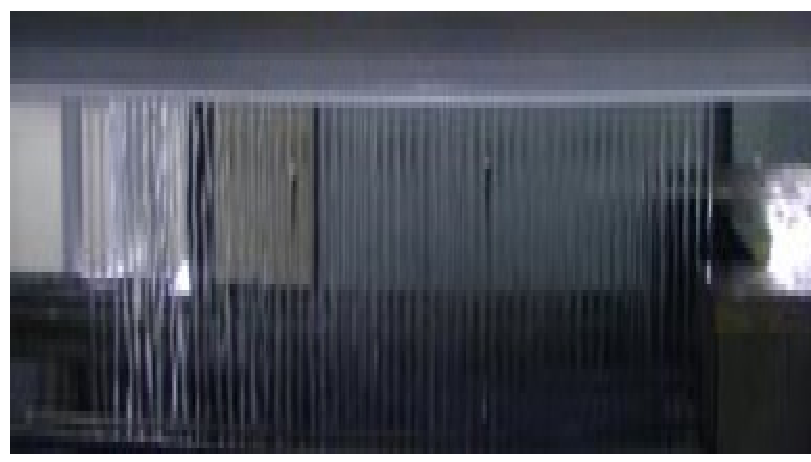

(a) Base model

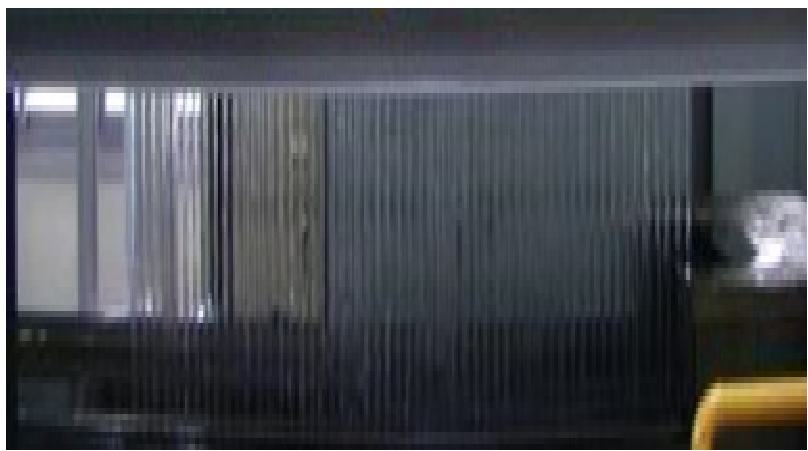

(b) Model with baffles

Figure 7. Photographs of the water distribution of the manifold: (a) Base model, (b) Model with the baffles.

The calculated results and the measured results for the distribution of flow matched relatively well.

Figure 7. represents two experimental results. The drive side of base model sprays imperfectly compared 
to the work side. The model with the baffles shows more even water jet columns than the base model.

\section{Conclusions}

An experimental and numerical study has been conducted to investigate the water distribution of the manifold for different Reynolds numbers of $4.2 \times 10^{5}$ and $8.4 \times 10^{5}$.

In the multi-phase model, the VOF model was suitable for finding the unbalanced area and the Eulerian model was appropriate for estimation of mal-distribution flow.

The distribution of the nozzle flow depends on the Reynolds number.

The installation of the baffles is effective to ease the flow distribution of a manifold under large flow condition.

\section{References}

1. P. Lee, H. Choi, S. Lee, ISIJ International 44, 704 (2004)

2. S.D. Cox, S.J. Hardy, D.J. Parker, Ironmaking and Steelmaking, 28, 363 (2001)

3. C. Devadas, I.V. Samarasekera, Ironmaking and Steelmaking, 13, 311 (1986)

4. J.F. Evans, I.D. Roebuck, H.R. Watkins, Iron and Steel Engineer, 70, 50 (1993)

5. X. Wu, R.L. Webb, Applied Thermal Engineering, 22, 1369, (2002)

6. S. Lalot, P. Florent, S. Lang, A. Bergles, Applied Thermal Engineering, 19, 847 (1999)

7. B.P. Rao, B. Sunden, S.K. Das, Journal of Heat Transfer, 127, 332 (2005)

8. B.P. Rao, B. Sunden, S.K. Das, International Journal of Heat and Mass Transfer, 49, 4966 (2006)

9. A. Marchitto, M. Fossa, G. Guglielmini, Experimental Thermal and Fluid Science, 33, 895 (2009)

10. E.R. Dario, L. Tadrist, J.C. Passos, Applied Thermal Engineering 59, 316 (2013)

11. Ansys Inc., Ansys fluent 14 user's guide (Ansys Inc., USA, 2011)

12. V.V Ranade, Computational Flow Modeling for Chemical Reactor Engineering, 5, (Academic Press, USA, 2002) 